\title{
In search of chondrocyte-specific antigen
}

\author{
ANNA OSIECKA-IWAN, PIOTR SKOPINSKI, DOROTA M. RADOMSKA-LESNIEWSKA, ANNA HYC
}

Department of Histology and Embryology, Medical University of Warsaw, Poland

\begin{abstract}
The purpose of this work was to establish, whether rat chondrocyte associated antigen, transmembrane Tmp 21 protein belonging to the 24 protein family may immunize rats and thus be included into the panel of immunogens potentially involved in cartilage pathology. For immunization of rats extract from cultured chondrocytes containing surface chondrocyte proteins suspended in incomplete Freund's adjuvant was used. Control animals were injected with incomplete Freund's adjuvant without chondrocyte extract. Morphological observations indicated that both in control and experimental animals occurred subperiosteal resorption of bone, suggesting that it arised as the response to adjuvant. In trachea, however, resorption of cartilage and inflammatory changes in the respiratory epithelium and lamina propria were present only in animals exposed to antigen. Unexpectedly, sera from immunized rats strongly reacted with other antigen, which we were able to identify by Western blot and protein sequencing as cartilage oligomeric matrix protein (COMP). COMP is attached to chondrocyte membrane by integrins and its presence in chondrocyte extract is not surprising. Antibody response to COMP raises a question whether the observed changes in tracheal cartilage and epithelium represent anti-COMP reaction or were caused by some other, no specified factors. COMP is used as the marker of osteoarthritis progression, but its role in polychondritis, cartilage pathology involving i.a. tracheal cartilage resorption remains unknown. Thus, our observations may serve as the starting point for future studies in this direction.
\end{abstract}

Key words: chondrocyte antigen, cartilage oligomeric matrix protein (COMP), transmembrane p24 trafficking protein 10 (TMP21).

(Centr Eur Immunol 2018; 43 (2): 132-138)

\section{Introduction}

Surface chondrocyte molecules of cartilage may serve as an example of sequestered antigens due to the presence of cartilage matrix preventing their contact with immune system [1-3]. Cartilage matrix contains the collagen network composed of types II, IX, and XI collagens, the large aggregating proteoglycan - aggrecan, and a large number of other molecules such as several small proteoglycans: biglycan, decorin, fibromodulin, matrilins, and cartilage oligomeric matrix protein (COMP) $[4,5]$. It is well established that collagen type II [6] and proteoglycans are able to stimulate immune response [7], but the antigenicity of chondrocytes and particularly the presence - or absence - of hypothetical tissue specific antigens remains as the no resolved puzzles of chondrocytes biology. Early work showed that isolated allogeneic chondrocytes after transplantation produce cartilage but also evoke the host immunization leading to rejection of new-formed tissue [8]. Demonstration of class I MHC expression by chondrocytes followed [9-12] subsequent publications indicated that chondrocytes are able to present antigens within MHC class II context $[13,14]$. In the more recent work, both human articular chondrocytes originating from patients who suffer from osteoarthritis (OA) and from healthy donors were shown to express both class I and class II MHC antigens [15]. In another study, however, authors found that cultured human OA chondrocytes expressed MHC class I but not MHC class II or positive co-stimulatory molecules, and failed to evoke both MHC class I and II mismatch allogeneic lymphocyte responses [16, 17].

Several authors suggested also that chondrocytes express chondrocyte-specific differentiation antigen. Langer et al. [18] have found that rats injected with syngeneic chondrocytes or cartilage shavings developed autoreactivity as assayed by leukocyte migration test. This result, according to the authors, indicated existence of a specific chondrocyte differentiation antigen. This conclusion was supported by studies demonstrating stimulation of proliferative response of rat lymphocytes in mixed lymphocyte-chondrocyte cultures not only by allogeneic but also by syngeneic chondrocytes $[19,20]$. The last observation was confirmed with rabbit [14], human, bovine [21] and rat chondrocytes [11, 22].

To obtain antibodies against chondrocyte-specific differentiation antigen rat chondrocytes were injected into rabbits. Chondrocytes reconstructed cartilage which within

Correspondence: Anna Hyc, PhD, Department of Histology and Embryology, Medical University of Warsaw, 5 Chalubinskiego St., 02-004 Warsaw, Poland, e-mail: ahyc@wum.edu.pl 
two weeks deteriorated, but the sensitized rabbits sera contained high titter of antichondrocyte cytotoxic antibodies which detected antigen with $\mathrm{M}_{\mathrm{r}}$ of $\sim 74$ and $\sim 23 \mathrm{kDa}$ in chondrocyte lysates. Only the latter remained after reduction in 2-mercaptoethanol. Neither antigen could be detected by this serum in lysates of fibroblasts, endotheliocytes, or thymocytes. Thus, it appeared that the antibodies of this serum reacted with chondrocyte-associated antigen (CAA), but the nature of the antigen remained unknown [23].

Since it is well established that chondrocytes grown in monolayer culture gradually decrease expression of typical matrix molecules such as collagen type II and aggrecan $[24,25]$, it seemed interesting to establish whether in such cultures expression of chondrocyte-specific differentiation antigen, or, as it was called in other papers, chondrocyte associated antigen (CAA) will also decline. Indeed, CAA expression in monolayer culture of rat chondrocytes gradually decreased and was not detected beyond the $96^{\text {th }}$ hour, as well as collagen type II and aggrecan [26]. After transfer of dedifferentiated chondrocytes into 3-dimensional culture CAA expression was restored, similarly as it was previously observed in case of collagen type II and aggrecan expression [26-28].

To identify the CAA proteins extracted from the surface of short-term chondrocyte cultures were sequenced and the protein corresponding to the $23-\mathrm{kDa}$ antigen was identified as transmembrane Tmp21 protein belonging to the p24 protein family [29]. These proteins mainly participate in the traffic between the endoplasmic reticulum (ER) and Golgi complex [30] but in some cells appear also in membranes of secretory vacuoles [31] and on cell surfaces $[32,33]$. Tmp21 extracted from chondrocytes was sialylated and ceased to bind sensitized rabbit serum after deglycosylation. Since the sialylated form of Tmp21 was not described in other tissues and seems appears to be specific for chondrocytes, it seems plausible to consider CAA as a chondrocyte differentiation antigen [26].

Since various chondrocyte antigens are supposed to play a role in pathogenesis of destructive joint diseases $[34,35]$ it seemed interesting to see whether crude CAA antigen extracted from chondrocytes may evoke immunological response in syngeneic system. Unexpectedly, in sera of sensitized rats antibodies reacting with Tmp21 glycosylated protein were not found, but strong response against cartilage oligomeric matrix protein (COMP) was detected. COMP or thrombospondin-5, is an ECM protein primarily present in cartilage but detected also in other connective tissues [36-38], where interacts with other extracellular matrix proteins such collagens and fibronectin. Since COMP may also bind to cell surface receptors [39], its presence in the extracts of chondrocyte cell membrane is not surprising, but such a strong immunostimulatory effect was, as far as we could establish, not previously noted.

\section{Material and methods}

\section{Animals}

3-5-day-old inbred Wistar Albino Glaxo (WAG) rats served as donors of cartilage for the isolation of chondrocytes. 3 month-old WAG male rats were used as a recipients of chondrocytes antigens extract. Animals were obtained from Animal Unit of the Medical University of Warsaw. The study was approved by the Animal Ethical Committee of the Medical University of Warsaw.

\section{Isolation and culture of chondrocytes}

Chondrocytes were isolated from the articular-epiphyseal cartilage complexes of 3-5-day-old rats. Cartilage fragments were cleaned from the surrounding tissues and digested using $0.25 \%$ collagenase, $0.05 \% \mathrm{DN}$-ase and $7 \mathrm{nM}$ N- $\alpha$-tosyl-L-lysyl chloromethyl ketone (all from Sigma-Aldrich Chemie, Diesenhofen, Germany) in $37^{\circ} \mathrm{C}$ for $3 \mathrm{~h}$ with constant stirring. Following isolation chondrocytes were filtered through $40 \mu \mathrm{m}$ mesh nylon cloth (Millipore Corporation, Bedford, MA 01730 USA), washed three times in RPMI (GIBCO BRL, Paisly, Scotland, UK), seeded into $35 \mathrm{~mm}$ Petri dishes $\left(5 \times 10^{6}\right.$ cells per dish) (Corning Inc., Corning, NY, USA) in $2 \mathrm{ml}$ of serumfree RPMI medium (GIBCO) supplemented with $1 \%$ of Antibiotic-Antimycotic Solution (Sigma) and cultured for $24 \mathrm{~h}$.

\section{Chondrocyte-associated antigens (CAAs) preparation}

After $24 \mathrm{~h}$ of culture, chondrocytes were shortly washed with distilled water and chondrocyte surface macromolecules were extracted for $30 \mathrm{~min}$, in room temperature with $2 \mathrm{M} \mathrm{NaCl}$ (Sigma). The suspension of surface proteins was desalted on PD-10 columns (Amersham Biosciences, Uppsala, Sweden), lyophilized and dissolved in $0.9 \%$ sodium chloride (Sigma).

\section{Protein determination}

One microliter of chondrocyte extract or extraction buffer (blank test) and $9 \mu$ deionized water were placed in a flat-bottomed 96-well plate, and $0.2 \mathrm{ml}$ of BCA protein assay reagent (Pierce, Rockford, IL, USA) was added to each well. The plate was incubated at $37^{\circ} \mathrm{C}$ for $30 \mathrm{~min}$. Protein concentration was determined spectrophotometrically at $550 \mathrm{~nm}$ (SLT Spectra Lab Instruments, Crailsheim, Germany)

\section{Immunization of recipients}

$200 \mathrm{mg}$ of chondrocyte-associated antigens (CAAs) in $150 \mu \mathrm{l}$ of $0,9 \% \mathrm{NaCl}$ was mixed with $150 \mu \mathrm{l}$ of incomplete Freund's adjuvant - IFA (Sigma) and injected subcutaneously in three sites at the base of the tails of 3 month-old 
male rats. Immunization procedure was repeated after 10 days using the same dose of chondrocyte extract. Animals were killed 2 weeks after the second immunization and blood from the heart (for the serum isolation), tracheas, synovial membranes and knee joints were taken for the further study. Control animals received only incomplete Freund's adjuvant (Sigma) according to the same protocol.

\section{Histological procedure}

Tracheas, synovial membranes and knee joints were fixed in phosphate-buffered formalin, $\mathrm{pH} 7.4$ and after paraffin mounting were sectioned at $6 \mu \mathrm{m}$. Slides were stained with hematoxylin and eosin (Sigma).

\section{Electrophoresis and Western blot}

Chondrocyte-associated antigens (CAAs) $(10 \mu \mathrm{g}$ of protein) mixed in sample buffer with 2-mercaptoethanol were separated by SDS-polyacrylamide gel electrophoresis (SDSPAGE; 12\% acrylamide) (Bio-Rad Laboratories, Hercules, CA, USA). Separated proteins were transferred onto PVDF membrane by semi-dry blotting at $25 \mathrm{~V}$ for 30 min using Trans-Blot SD apparatus (Bio-Rad). Serum from chondrocyte extract-immunized rats diluted $1: 120$ or commercial antibodies against COMP diluted 1 : 2000 (GeneTex, Hsinchu City 300, Taiwan) served as the primary antibodies. After $1 \mathrm{~h}$ of incubation rabbit anti-rat biotinylated immunoglobulins or biotinylated $\mathrm{F}(\mathrm{ab}$ ')2 fragments of swine anti-rabbit immunoglobulins (Dako A/S, Glostrup, Denmark) secondary antibodies was added respectively. Actin used as the internal control was detected with mouse monoclonal anti- $\beta$-actin antibody (Sigma) followed by biotinylated goat anti-mouse immunoglobulins (Dako). Antibody binding was demonstrated by an amplified alkaline phosphatase detection system (Bio-Rad). The relative molecular weight of the antigens was calculated using GelWorks 1D Intermediate software (Bio-Rad) in relation to the pre-stained SDS-PAGE (low range) standard (Bio-Rad). The second polyacrylamide gel after protein electrophoresis was stained with Coomassie blue and the band corresponding to the band detected by serum from CAAs-immunized rats was cut off for protein sequence analysis.

\section{Protein sequence analysis}

Analysis and data processing was done on the commercial basis in Mass Spectrometry Laboratory, Institute of Biochemistry and Biophysics of the Polish Academy of Sciences, Warsaw, Poland according to the following procedure.

\section{Trypsin digestion}

Gel slices were subjected to standard "in-gel digestion" procedure during which proteins were reduced with $100 \mathrm{mM}$ 1,4-dithiothreitol (Sigma) (for $30 \mathrm{~min}$ at $56^{\circ} \mathrm{C}$ ), alkylated with iodoacetamide ( $45 \mathrm{~min}$ in darkroom at room temperature) and digested overnight with trypsin (sequencing Grade Modified Trypsin - Promega V5111). Resulting peptides were eluted from gel with $0.1 \%$ trifluoroacetic acid (TFA) and $2 \%$ acetonitrile (ACN) (Sigma).

\section{Mass spectrometry}

Peptide mixtures were separated by liquid chromatography prior to molecular mass measurements on Orbitrap Velos mass spectrometer (Thermo Electron Corp., San Jose, CA). Peptide mixture was applied to RP-18 precolumn (nanoACQUITY Symmetry® C18 - Waters 186003514) using water containing $0.1 \%$ TFA as mobile phase and then transferred to nano-HPLC RP-18 column (nanoACQUITY BEH C18 - Waters 186003545) using an acetonitrile gradient (0$60 \% \mathrm{ACN}$ in $70 \mathrm{~min}$ ) in the presence of $0.05 \%$ formic acid with the flow rate of $150 \mathrm{nl} / \mathrm{min}$. Column outlet was directly coupled to the ion source of the spectrometer working in the regime of data dependent MS to MS/MS switch. A blank run ensuring lack of cross contamination from previous samples preceeded each analysis.

\section{Data processing}

Acquired raw data were processed by Mascot Distiller followed by database search with Mascot program (Matrix Science, London, UK, 8-processor on-site license) against NCBInr (version 20100203). Search parameters for precursor and product ions mass tolerance were $40 \mathrm{ppm}$ and $0.8 \mathrm{Da}$,
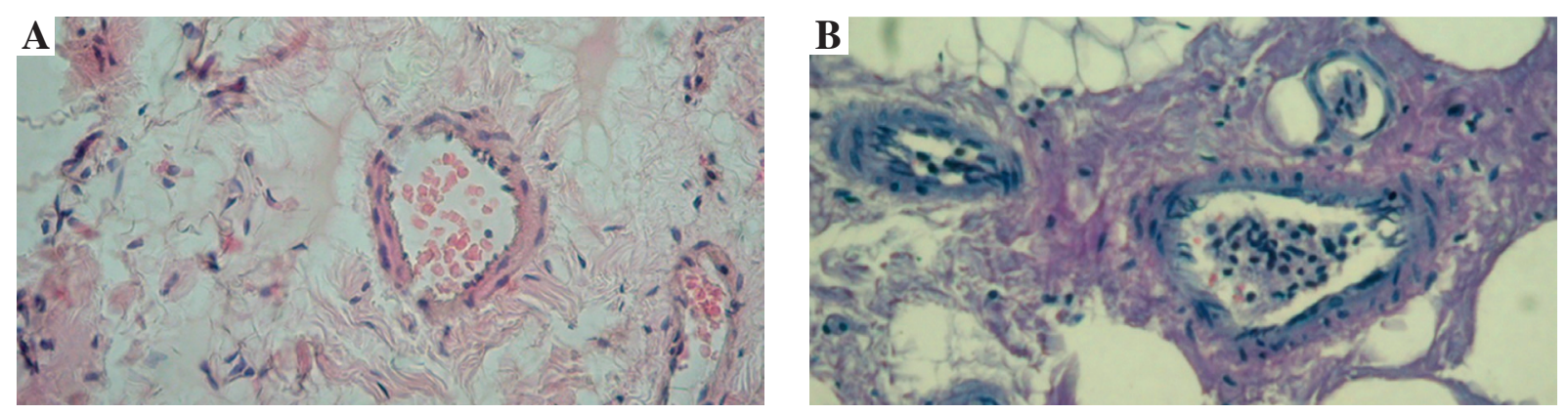

Fig 1. A) Synovial membrane from incomplete Freund's adjuvant (IFA) injected rat. B) Synovial membrane of rat after IFA-antigen administration - increased number of leukocytes in blood vessels. H\&E 400x 


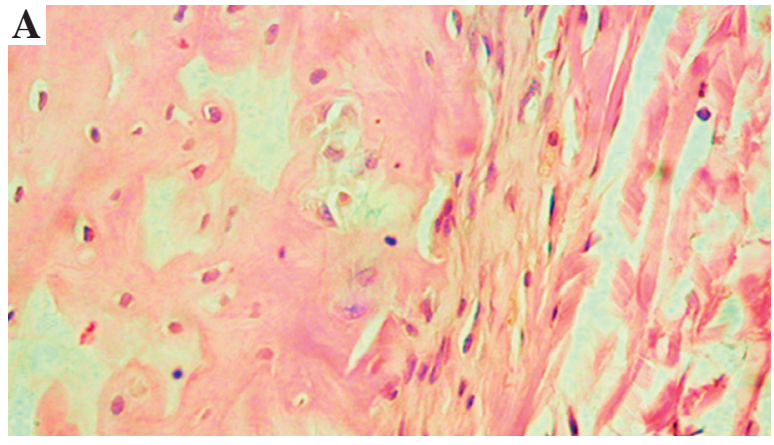

Fig. 2. A) Bone from control (intact) rat. B) Bone after administration of IFA only. C) Bone after administration of IFA with antigen. A-C area of bone - periosteum contact, in all groups subperiosteal resorption of bone with participation of osteoclasts was observed. H\&E 200×

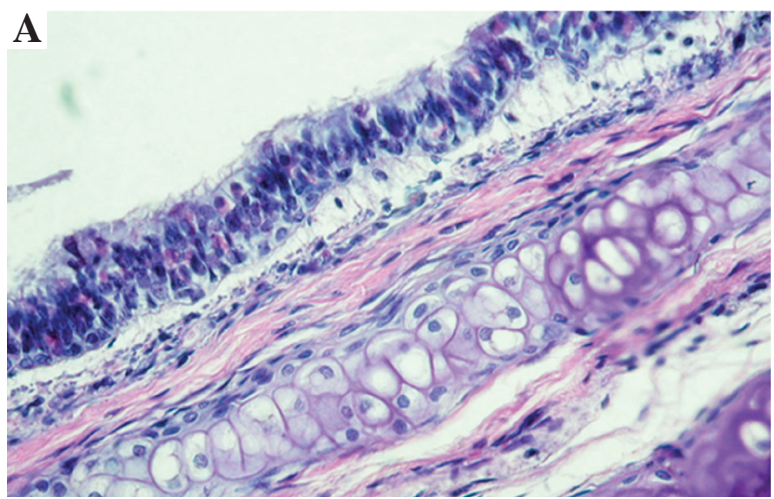

Fig. 3. A) Trachea from rat receiving IFA only. Respiratory epithelium appears unchanged, cells display distinct cilia. B) Tracheal respiratory epithelium after IFA-antigen administration. Epithelium is heavily infiltrated by leukocytes and disappearance of cilia is observed. H\&E 200x. C) Cartilage of tracheal ring after IFA-antigen administration. Cartilage is peripherally resorbed by infiltrating cells (arrow). H\&E 400x

respectively, with allowance made for one missed trypsin cleavage, the following fixed modifications: cysteine carbamidomethylation and allowed variable modification: oxidation (M). Peptides with Mascot Score exceeding the threshold value corresponding to $<5 \%$ false positive rate, calculated by Mascot procedure, were considered to be positively identified.
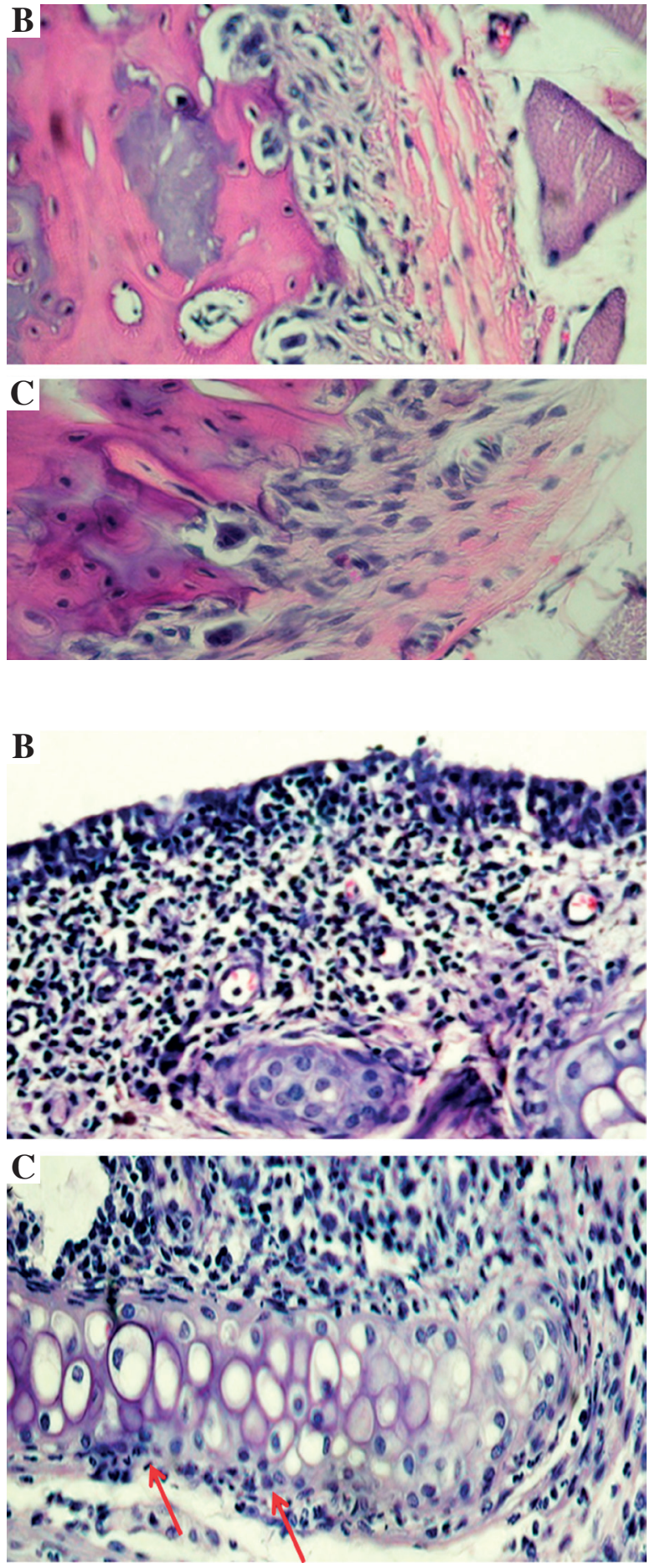

\section{Results}

\section{Histological evaluation}

In control (incomplete Freund's adjuvant - IFA alone) and experimental synovial membranes in comparison with pristine samples the most significant change was the pres- 


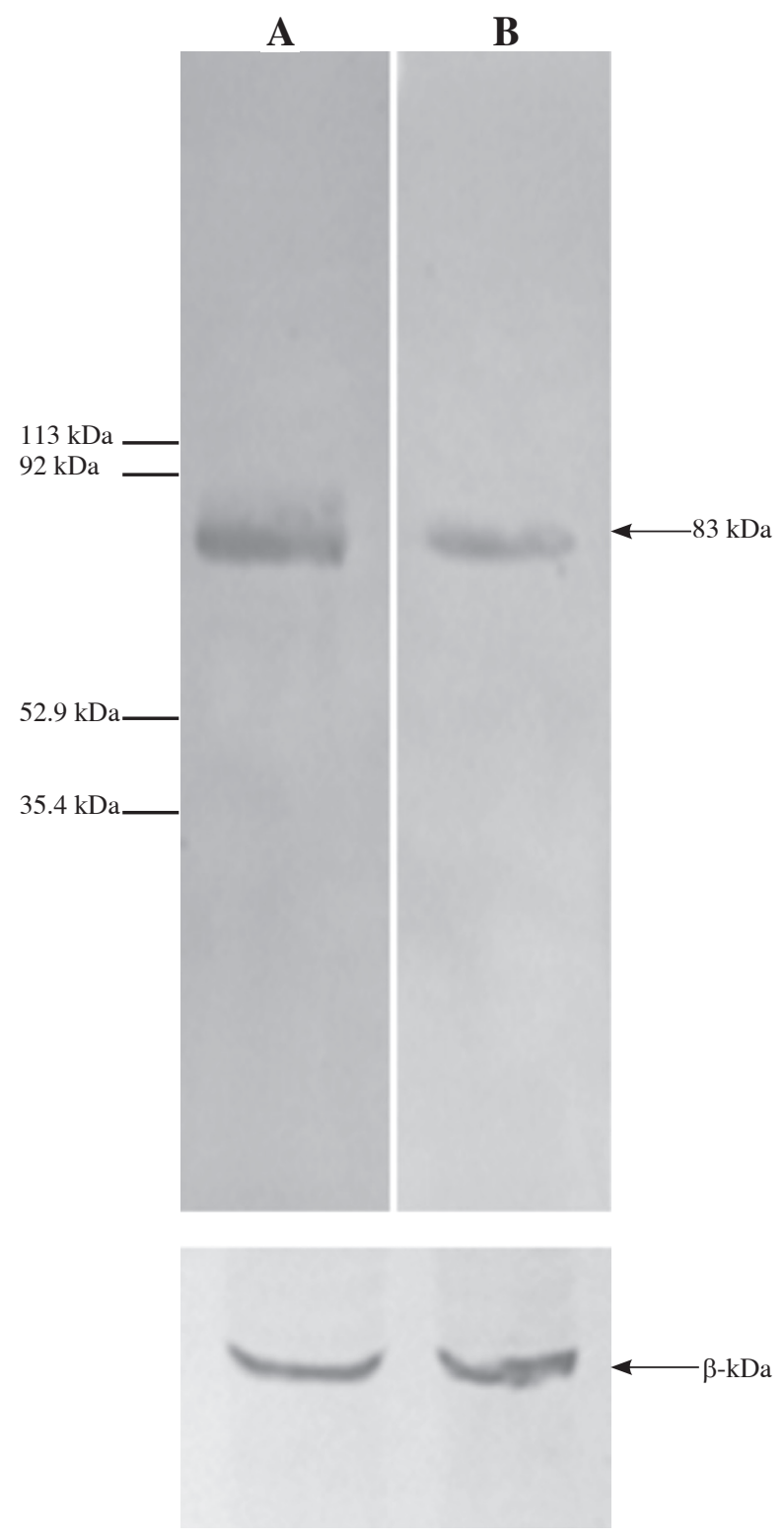

Fig. 4. Western blot analysis of chondrocyte extract. A) Commercial antibodies against COMP and B) sera from rats immunized with chondrocyte antigens in incomplete Freund's adjuvant detected antigen with $\mathrm{M}_{\mathrm{r}}$ of $\sim 83 \mathrm{kDa}$, corresponding to COMP. Actin was used as an internal control

ence of increased number of leukocytes in blood vessels (Fig. 1). Changes in bone were observed in epiphyses of tibiae close to the articular surface. Both in IFA and adjuvant IFA-antigen specimens occurred marked, subperiosteal resorption with participation of osteoclasts (Fig. 2) while cartilage was not affected. Cartilage forming the tracheal rings was, however, peripherally resorbed by infiltrating cells but only in animals receiving antigen. Respiratory epithelium lining tracheae was not affected in animals ex- posed to IFA alone, but after antigen administration was heavily infiltrated by leukocytes which in many places replaced epitheliocytes. Cilia were usually absent (Fig. 3).

\section{Western blot}

Sera from rats immunized with chondrocyte antigens in incomplete Freund's adjuvant and commercial antibodies against COMP detected in chondrocyte extract antigen with $\mathrm{M}_{\mathrm{r}}$ of $\sim 83 \mathrm{kDa}$ (Fig. 4).

\section{Protein sequence determination}

Mass spectrometry data processing of a proteins present in the band reacting with sensitized rabbit serum was repeated six times, and all determinations gave similar results. Results of one sequencing are given in Table 1 . The studied protein had a mass of 82611 Daltons and Mascot score 697 calculated on the basis of six matching peptides with ions score in the range from 55 to 108 (Table 1). Protein sequence coverage was $19 \%$ and revealed as the best matched protein the cartilage oligomeric matrix protein (COMP).

\section{Discussion}

Results of administration of proteins extracted from chondrocyte membranes yielded unexpected results. In the sera of injected rats no antibodies against transmembrane Tmp21 protein were detected but Western blot analysis disclosed the presence of antibodies reacting with antigen with molecular weight of about $83 \mathrm{kDa}$ in the reduced state. The same antigen was also detected by anti-COMP commercial antibodies. The identification of this antigen as COMP was also confirmed by sequence analysis. The morphological analysis of results was complicated by the use of IFA in which chondrocyte surface proteins were suspended. The use of adjuvants is the common procedure to increase immune response to antigens [40], but adjuvant itself may also cause untoward response affecting various tissues. Effects of incomplete Freund's adjuvant (IFA) were found to be related to the strain of rats. Injected Lewis rats did not develop clinical signs of arthritis while administration of IFA to DA (dark agouti) rats evoked strong arthritogenic response [41]. The WAG rats used in this work also proved to react strongly to free of antigen IFA administration and changes in joint tissues were similar to those in rats injected with antigen. The main morphological differences between antigen free and antigen containing adjuvant injected animals was the presence of heavy infiltrations in the tracheal epithelium and peripheral resorption of cartilage of tracheal rings. Presence of anti-COMP antibodies in immunized rats directed our attention on the relation between COMP and cartilage. In healthy cartilage COMP is attached to chondrocyte cell membrane via integrin receptors $[39,42]$ and is also present in the pericellular 
Table 1. Amino acid sequences of peptides present in COMP found by Mascot search

\begin{tabular}{cccc}
\hline Number & $\begin{array}{c}\text { Localization in protein } \\
\text { (start-end) }\end{array}$ & Peptide sequences & Possible modifications \\
\hline 1 & $36-48$ & R.ELQETNAALQDVR.E & \\
\hline 2 & $200-216$ & R.GSFQCGPCQPGFVGDQR.S & 2-Carbamidomethyl (C) \\
\hline 3 & $239-245$ & K.ADCILER.D & \\
\hline 4 & $484-495$ & R.LVPNPGQmidomethyl (C) \\
\hline 5 & $515-530$ & K.IDVCPENAEVTLTDFR.A & Carbamidomethyl (C) \\
\hline 6 & $640-649$ & K.SSTGPGEQLR.N & \\
\hline
\end{tabular}

space and territorial and interterritorial matrix [43]. The release of COMP from cartilage occurs in OA [44, 45] and high serum levels of COMP are associated with the OA progression [46-49]. Autoantibodies to COMP were also detected in patients with rheumatoid arthritis (RA) [50]. Induction of arthritis by immunization of rats with COMP emulsified in IFA was also reported. Morphological changes were accompanied by the appearance of anti-COMP antibodies in rat's sera [51].

Release of COMP from cartilage occurs also in polychondritis and may involve tracheobronchial tree causing respiratory tract symptoms. Immune-mediated damage can spread to noncartilaginous tissues that are rich in proteoglycans, such as that of the eyes, the inner ear, the heart, blood vessels, and the kidney, but as far as we could establish, changes in the tracheal epithelium were not reported [52-55]. In relation to experimental relapsing polychondritis Hansson and Holmdahl [56] stress that only joints were affected in COMP immunized rats as reported by Carlsén et al. [51]. In our work after administration of proteins extracted from articular chondrocytes in IFA the tracheal cartilages were affected. Since, however, the COMP used in their studies was purified from rat chondrosarcoma tissue and in this work was extracted together with other proteins from chondrocyte cell membrane, the possible role of COMP immunization in polychondritis development requires further studies. We also would like to stress, that the lack of antibodies against transmembrane Tmp21 protein belonging to the $\mathrm{p} 24$ protein family in immunized rats does not disqualify it as the tissue specific antigen, but rather suggests that although it is strongly immunogenic in rabbits may not be involved to the same degree in cartilage pathology in other species.

The authors declare no conflict of interest.

\section{References}

1. Bacsich P, Wyburn GM (1947): The significance of the mucoprotein content on the survival of homografts of cartilage and cornea. Proc R Soc Edin Sect B 62: 321-327.
2. Heyner $S$ (1969): The significance of the intercellular matrix in the survival of cartilage allografts. Transplantation 8: 666-677.

3. Heyner S (1973): The antigenicity of cartilage grafts. Surg Gynec Obstet 135: 298-305.

4. Otero M, Goldring MB (2007): Cells of the synovium in rheumatoid arthritis Chondrocytes. Arthritis Res Ther 9: 220.

5. Gao Y, Liu S, Huang J, et al. (2014): The ECM-Cell Interaction of Cartilage Extracellular Matrix on Chondrocytes. Biomed Res Int 2014: 648459.

6. Krco CJ, Pawelski J, Harders J, et al. (1996): Characterization of the antigenic structure of human type II collagen. J Immunol 156: 2761-2768.

7. Misják P, Bôsze S, Horváti K, et al. (2013): The role of citrullination of an immunodominant proteoglycan (PG) aggrecan T cell epitope in BALB/c mice with PG-induced arthritis. Immunol Lett 152: 25-31.

8. Moskalewski S, Kawiak J, Rymaszewska T (1966): Local cellular response evoked by cartilage formed after auto- and allogeneic transplantation of isolated chondrocytes. Transplantation 4: $572-581$.

9. Elves MW (1974): A study of the transplantation antigens on chondrocytes from articular cartilage. J Bone Joint Surg 56-B: 178-185.

10. Malseed ZM, Heyner S (1976): Antigenic profile of the rat chondrocytes. Arthritis Rheum 19: 223-231.

11. Lance EM (1989): Immunological reactivity towards chondrocytes in rat and man: relevance to autoimmune arthritis. Immunol Lett 21: 63-73.

12. Lance EM, Kimura LH, Manibog CN (1993): The expression of major histocompatibility antigens on human articular chondrocytes. Clin Orthop Rel Res 291: 266-282.

13. Bujia J, Alsalameh S, Sittinger M, et al. (1994): Antigen presenting cell function of class II positive human nasal chondrocytes. Acta Otolaryngol 114: 75-79.

14. Tiku ML, Liu SW, Weaver CW, et al. (1985): Class II histocompatibility antigen-mediated immunologic function of normal articular chondrocytes. J Immunol 135: 2923-2928.

15. O'Sullivan NA, Ibusuki S, Yaremchuk MJ, Randolph MA (2006): Antigenicity of allogeneic human chondrocytes for tissue engineering. 52 ${ }^{\text {nd }}$ Annual Meeting of the Orthopaedic Research Society. Paper No: 1446.

16. Abe S, Nochi H, Ito H (2016): Alloreactivity and immunosuppressive properties of articular chondrocytes from osteoarthritic cartilage. J Orthop Surg (Hong Kong) 24: 232-239.

17. Lohan P, Treacy O, Lynch K, et al. (2016): Culture expanded primary chondrocytes have potent immunomodulatory properties and do not induce an allogeneic immune response. Osteoarthritis and Cartilage 24: 521-533. 
18. Langer F, Gross AE, Greaves MF (1972): The auto-immunogenicity of articular cartilage. Clin Exp Immunol 12: 31-37.

19. Gertzbein SD, Lance EM (1976): The stimulation of lymphocytes by chondrocytes in mixed culture. Clin Exp Immunol 24: 102-109.

20. Gertzbein SD, Tait JH, Devlin SR, Argue S (1977): The antigenicity of chondrocytes. Immunology 33: 141-145.

21. Glant T, Mikecz K (1986): Antigenic profiles of human, bovine and canine articular chondrocytes. Cell Tissue Res 244: 359-369.

22. Osiecka-Iwan A, Hyc A, Moskalewski S (1999): Immunosuppression and rejection of cartilage formed by allogeneic chondrocytes in rats. Cell Transpl 8: 627-636.

23. Osiecka-Iwan A, Hyc A, Jozwiak J, et al. (2003): Transplants of Rat Chondrocytes Evoke Strong Humoral Response Against Chondrocyte-Associated Antigen in Rabbits. Cell Transplant 12: 389-398.

24. Mayne R, Vail MS, Maine PM, Miller EJ (1976): Changes in type of collagen synthesis as clones of chick chondrocyte grow and eventually lose division capacity. Proc Natl Acad Sci USA 73: 1674-1678.

25. Schlitz JR, Mayne R, Holtzer H (1973): The synthesis of collagen and glycosaminoglycans by dedifferentiated chondrocytes in culture. Differentiation 1: 97-108.

26. Osiecka-Iwan A, Hyc A, Niderla-Bielinska J, Moskalewski S (2008): Chondrocyte-associated antigen and matrix components in a 2- and 3-dimensional culture of rat chondrocytes. Mol Med Rep 1: 881-887.

27. Benya PD, Shaffer JD (1982): Dedifferentiated chondrocytes reexpress the differentiated collagen phenotype when cultured in agarose gels. Cell 30: 215-224.

28. Bonaventure J, Kadhom N, Cohen-Solal L, et al. (1994): Reexpression of cartilage-specific genes by dedifferentiated human articular chondrocytes cultured in alginate beads. Exp Cell Res 212: 97-104.

29. Osiecka-Iwan A, Niderla-Bielinska J, Hyc A, Moskalewski S (2014): Rat chondrocyte-associated antigen identified as sialylated transmembrane protein Tmp21 belonging to the $\mathrm{p} 24$ protein family. Calcif Tissue Int 94: 348-352.

30. Strating JR, van Bakel NH, Leunissen JA, Martens GJ (2009): A comprehensive overview of the vertebrate p24 family: identification of a novel tissue-specifically expressed member. Mol Biol Evol 26: 1707-1714.

31. Hosaka M, Watanabe T, Yamauchi Y, et al. (2007): A subset of $\mathrm{p} 23$ localized on secretory granules in pancreatic beta-cells. J Histochem Cytochem 55: 235-245.

32. Füllenkrug J, Suganuma T, Tang BL, et al. (1999): Localization and recycling of gp27 (hp24c3): complex formation with other p24 family members. Mol Biol Cell 10: 1939-1955.

33. Blum R, Lepier A (2008): The luminal domain of 23 (Tmp21) plays a critical role in p23 cell surface trafficking. Traffic 9: 1520-1550.

34. Alsalameh S, Mollenhauer J, Hain N, et al. (1990): Cellular immune response towards human articular chondrocytes. Arthrit. Rheumat 33: 1477-1486.

35. Sabbatini AC, Tacchetti S, Tommasi F, et al. (2003): Use of human chondrocyte cell cultures to identify and characterize reactive antibodies in rheumatoid arthritis sera. Clin Exp Rheumatol 21: 587-592.

36. Agarwal P, Zwolanek D, Keene DR, et al. (2012): Collagen XII and XIV, new partners of cartilage oligomeric matrix protein in the skin extracellular matrix suprastructure. J Biol Chem 287: 22549-22559.
37. Wang L, Zheng J, Du Y, et al. (2010): Cartilage oligomeric matrix protein maintains the contractile pheno-type of vascular smooth muscle cells by interacting with alpha(7)beta(1) integrin. Circ Res 106: 514-525.

38. Müller G, Michel A, Altenburg E (1998): COMP (cartilage oligomeric matrix protein) is synthesized in ligament, tendon, meniscus, and articular cartilage. Connect Tissue Res 39: 233244.

39. Acharya C, Yik JH, Kishore A, et al. (2014): Cartilage oligomeric matrix protein and its binding partners in the cartilage extracellular matrix: Interaction, regulation and role in chondrogenesis. Matrix Biology 37: 102-111.

40. McKee AS, Munks MW, Marrack P (2007): How Do Adjuvants Work? Important Considerations for New Generation Adjuvants. Immunity 27: 687-690.

41. Kleinau S, Erlandsson H, Holmdahl R, Klareskog L (1991): Adjuvant oils induce arthritis in the DA rat. I. Characterization of the disease and evidence for an immunological involvement. J Autoimmun 4: 871-880.

42. Rock MJ (2010): Cartilage oligomeric matrix protein promotes cell attachment via two independent mechanisms involving CD47 and $\alpha$ V $\beta 3$ integrin. Mol Cell Biochem 338: 215-224.

43. Koelling S, Clauditz TS, Kaste M, Miosge N (2006): Cartilage oligomeric matrix protein is involved in human limb development and in the pathogenesis of osteoarthritis. Arthritis Res Ther 8: R56.

44. Mansson B, Carey D, Alini M, et al. (1995): Cartilage and bone metabolism in rheumatoid arthritis. Differences between rapid and slow progression of disease identified by serum markers of cartilage metabolism. J Clin Invest 95: 1071-1077.

45. DiCesare PE, Carlson CS, Stolerman ES, et al. (1996): Increased degradation and altered tissue distribution of cartilage oligomeric matrix protein in human rheumatoid and osteoarthritic cartilage. J Orthop Res 14: 946-955.

46. Wisłowska M, Jabłońska B (2005): Serum cartilage oligomeric matrix protein (COMP) in rheumatoid arthritis and knee osteoarthritis. Clin Rheumatol 24: 278-284.

47. Posey KL, Hecht JT (2008): The role of cartilage oligomeric matrix protein (COMP) in skeletal disease. Curr Drug Targets 9: 869-877.

48. Williams FM, Spector TD (2008): Biomarkers in osteoarthritis. Arthritis Res Ther 10: 101.

49. Verma P, Dalal K (2013): Serum cartilage oligomeric matrix protein (COMP) in knee osteoarthritis: a novel diagnostic and prognostic biomarker. J Orthop Res 31: 999-1006.

50. Weitoft T, Larsson A, Saxne T, et al. (2017): Pentraxin 3 in serum and synovial fluid of patients with rheumatoid arthritis with and without autoantibodies. Scand J Rheumatol 46: 346-352.

51. Carlsén S, Hansson AS, Olsson H, et al. (1998): Cartilage oligomeric matrix protein (COMP)-induced arthritis in rats. Clin Exp Immunol 114: 477-484.

52. Molina JF, Espinoza LR (2000): Relapsing polychondritis. Baillieres Best Pract Res Clin Rheumatol 14: 97-109.

53. Chopra R, Chaudhary N, Kay J (2013): Relapsing polychondritis. Rheum Dis Clin N Am 39: 263-276.

54. Sharma A, Gnanapandithan K, Sharma K, Sharma S (2013): Relapsing polychondritis: a review. Clin Rheumatol 32: 1575-1583.

55. Hirayama K, Iwanaga N, Izumi Y, et al. (2016): A Case of Relapsing Polychondritis Initiating with Unexplained Fever. Case Rep Med 2016: 9462489.

56. Hansson A-S, Holmdahl R (2002): Cartilage-specific autoimmunity in animal models and clinical aspects in patients - focus on relapsing polychondritis. Arthritis Res 4: 296-301. 